\title{
An Integrated Model of a Fixture-Workpiece System for Surface Quality Prediction
}

\author{
Y. G. $\mathrm{Liao}^{1}$ and S. J. Hu${ }^{2}$ \\ ${ }^{1}$ NA Car Group, General Motors Corporation, MC 480-305-2000, 5330 Clearview Drive, Troy, Michigan 48098, USA; and \\ ${ }^{2}$ Department of Mechanical Engineering and Applied Mechanics, 2350 Hayward Street, The University of Michigan, Ann Arbor, \\ Michigan 48109, USA
}

Surface quality is a major factor affecting the performance of a component. The machined surface quality is strongly influenced by the external loads during the fixturing and machining processes. In machining process development, it is highly desirable to predict the quality of a machined surface. For this purpose, an integrated finite element analysis (FEA) model of the entire fixture-workpiece system is developed to investigate the influence of clamping preload and machining force on the surface quality of the machined workpiece. The effects of fixture and machine table compliance (from experimental data), and the workpiece and its locators/clamps contact interaction, and forced vibration, on the machined surface quality are taken into account. This simulation model provides a better understanding of the causes of surface error and a more realistic prediction of the machined surface quality. The deck face of a V-type engine block subjected to fixture clamping and a face milling operation is given as an example. A comparison between the simulation result and experimental data shows a reasonable agreement.

Keywords: Clamping preload; Fixture; Forced vibration, Machining force, Surface error

\section{Introduction}

The quality of a machined surface is becoming increasingly critical for satisfying the demands for superior component performance, durability, and reliability. Structural components for aerospace and automotive industries are being subjected to more severe conditions of contact fatigue, stress, and temperature, and section size is being reduced in response to the goal of weight reduction. Thus, the surface quality of a component, which is strongly governed by external loads and fixturing during the manufacturing process, has an ever increasing influ-

Correspondence and offprint requests to: Y. G. Liao, General Motors Corporation, 5330 Clearview Drive, Troy, MI 48098, USA. E-mail: geneliao@ameritech.net ence on its performance. A model capable of analysing the causes of machined surface error and of realistically predicting the surface quality of a workpiece, subjected to fixturing and machining loads, is highly desired. Such predicted quality not only assists manufacturing engineers in determining a better process, and better fixturing, and tooling, but also provides advice to design engineers in product development.

The external loads, fixture stiffness, and contact interaction between a workpiece and its locators/clamps, and fixtureworkpiece dynamic stiffness, have a considerable impact on the final part accuracy. Therefore, an applicable analytical system to predict the quality of a machined surface should have workpiece structure, clamping preloads, machining forces, fixture compliance, contact interaction, and fixture-workpiece dynamic response included in the analysis. However, most of the prior research has not considered these factors simultaneously in machined surface quality prediction. A considerable amount of work has been carried out on the prediction of machining force and static analysis of a fixture. For example, Lee and Haynes [1], Menassa and DeVries [2], Subramani et al. [3], Kakade and Chow [4], Trappey et al. [5], Melkote et al. [6], and $\mathrm{Gu}$ et al. [7] used finite-element analysis (FEA) to predict the workpiece deflection due to external loads (clamping or machining forces), but did not consider the effects of fixture compliance and contact interaction. Chandra et al. [8] took clamping preload, clamping sequence, machining force, fixture compliance, and contact interaction into account for predicting workpiece deflection. They applied a gap-element along with a weak spring to represent the contact behaviour, which was only a 2D node-to-node approach. The other constraint of their model was that the dynamic response of the fixture-workpiece system was ignored. Thus far, no previous worker has treated machine tool, fixture, and workpiece as an integrated system.

This paper develops a model aimed at predicting the machined surface quality of a fixtured workpiece. In this more sophisticated model, the external loads, fixture compliances, workpiece and its locators/clamps surface-based contact interaction, and dynamic stiffness of the fixture-workpiece system, are taken into account. The stiffness of the fixture components 
and machine table is acquired from experiments. The FEA codes ABAQUS [9] and NASTRAN [10] along with DMAP (direct matrix application program) and Fortran programs are used to simulate a production engine block subject to fixture clamping and a face milling operation. ABAQUS's surfacebased contact pair is applied to model the fixture-workpiece contact interaction, and NASTRAN is used to obtain the dynamic stiffness of the fixture-workpiece system. The simulation procedure developed can be used to obtain a better understanding of the causes of surface error, and in particular, to determine the effects of clamping preloads, machining forces, and forced vibrations of the fixture-workpiece system, on the surface error. Experimental data is presented for comparison with the simulation result.

\section{Model Integration}

An integrated model is constructed by combining an FEA model of a local region of a fixture-workpiece system (workpiece and its locators/clamps) with the experimental stiffness of fixture bases and the machine table. In this integrated model, a superelement is also applied to model a real production workpiece of large dimension and complex geometry. The purpose of an integrated model is to provide effective modelling and computing for the entire fixture-workpiece system.

\subsection{Workpiece FEA Model}

The element type applied to model the workpiece is a solid brick element. Any edge dimension of an element is not larger than $10 \mathrm{~mm}$, leading to a sufficient number of nodes and elements. Although a fine-element mesh, or high mesh density, can represent the workpiece more realistically, the effort of computation increases significantly for a large complex part. This difficulty is solved by applying a superelement to model those subcomponents (or substructures) of a workpiece, which are not the machining and fixturing features of interest. In this way, the actual size of the FEA model is reduced.

\subsection{Locator/Clamp Compliance}

The locator is assumed to have a spherical tip, and the area of contact is assumed to be small compared with the radius of the fixture element. Considering a spherical tip locator contacting with a flat surface of the workpiece, the deflection at the contact point is given by [11].

$$
y_{l}=\left[0.465 \frac{P^{2}}{r_{l}}\left(\frac{1}{E_{w p}}+\frac{1}{E_{l}}\right)^{2}\right]^{1 / 3}
$$

where $y_{l}$ is the deflection of the locator, $E_{w p}$ and $E_{l}$ are the modulus of elasticity of workpiece and locator, respectively, $P$ is the total load, and $r_{l}$ is the sphere radius. This equation provides a force-displacement relationship for a compliant locator. It also indicates that the spring stiffness is nonlinear for a locator.
In a general fixturing system, the stiffness of a clamp is larger than that of a locator. The stiffness of the clamp was measured experimentally by [12]. The force-displacement relationship of a clamp has been found to be linear, i.e. the spring stiffness of the clamp is linear.

\subsection{Surface-Based Contact Modelling}

The contact interaction between the workpiece and its locators/clamps is modelled as a surface-based contact pair and is simulated as the small-scale sliding of 3D deformable bodies against each other. In this approach, both the rigid-body motion of the workpiece and the local elastic deformation of the fixture-workpiece interface are considered. The procedure of this surface-based contact approach is illustrated in [13].

\subsection{Stiffness of the Fixture Base}

A fixture base is an assembly for fixture-supporting structures. The stiffness of a fixture base is an important performance measurement of fixture design. Hence, this stiffness data is essential for the prediction of workpiece quality. The fixture stiffness is defined as the ratio of a unit external force to the total deformation of the fixture component in a sensitive direction of machining accuracy [14]. This stiffness can be static if the external load is static (such as clamping force), and dynamic if the external load is dynamic (such as machining force).

To address a practical problem, a modular machining fixture for an automotive engine cylinder block is considered. Figure 1 shows the basic assembly unit, where the columns and locating blocks are welded vertically to a baseplate. The locating pads and pins are bolted to these locating blocks. Then this fixture assembly is fastened to the machine table. When an external force $(F)$ is applied to the upper clamping plate in a vertical direction, the deflection is measured as $d s$ in the vertical direction. Similarly, the deflections in other directions can be obtained. The same measurement set-up is also applied to the locating block, so that the stiffness of this type of fixture can be computed.

\subsection{Stiffness of the Machine Table}

A machine table rides on the bedways and is powered in the translational directions by a hydraulic cylinder, screw, or rackand-pinion drive system. The table usually has a T-slot arrangement or a bolt-hole pattern for holding fixtures. Additionally, many machine-tool tables provide for an indexing function of the fixture-workpiece structure. Owing to this configuration, the tilt and turning deflections of a machine table are two major measurements in CNC machine tool performance evaluation. Figures $2(a)$ and $2(b)$ illustrate these measurements from several machine tool makers [15]. 


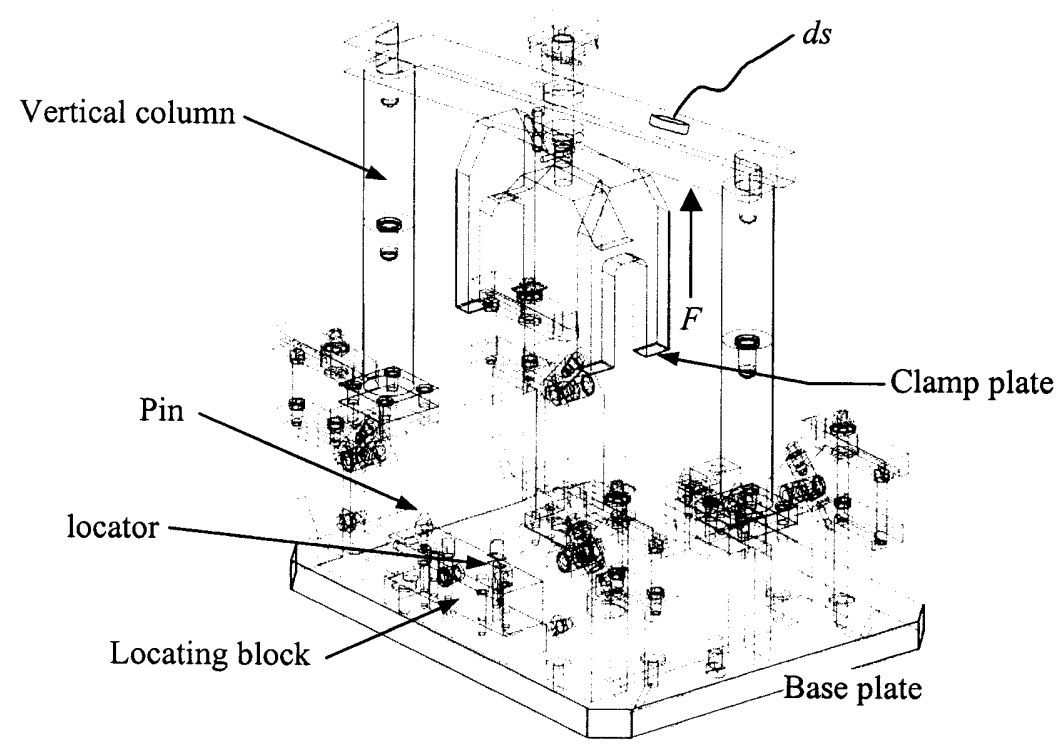

Fig. 1. Stiffness measurement of a modular fixture.

(a)

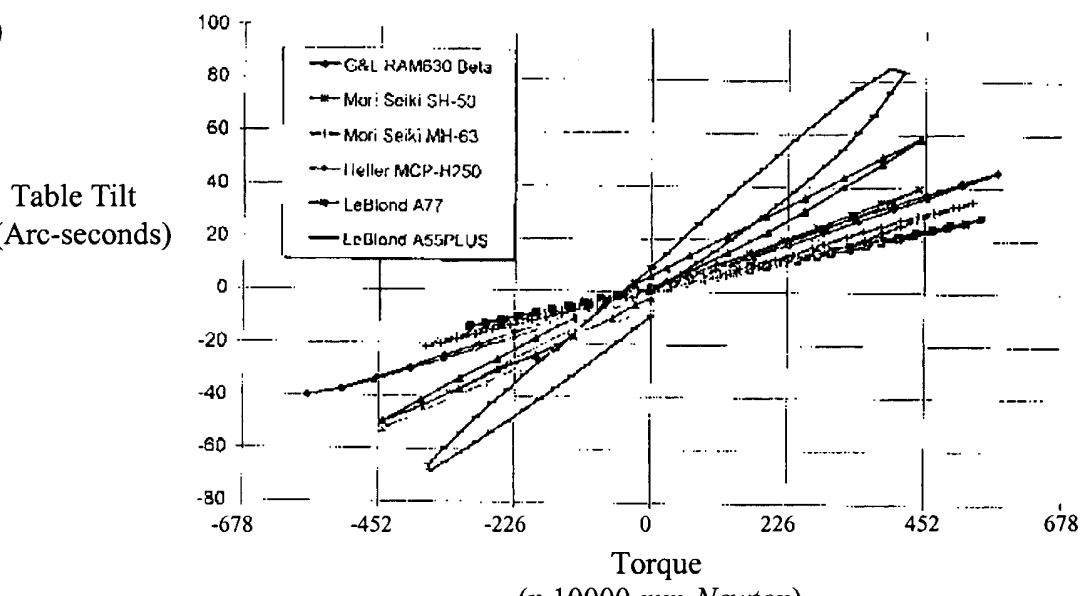

(x 10000 mm-Newton)

(b)

Table Tilt

(Arc-seconds)

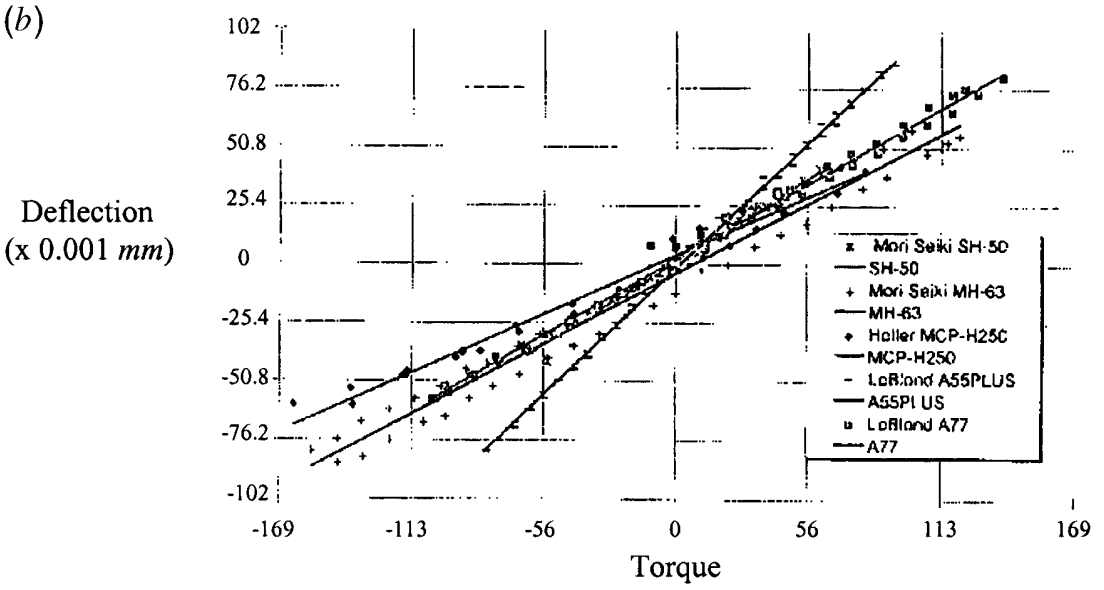

(x 10000 mm-Newton)

Fig. 2. Measurement of $(a)$ machine table tilt, and $(b)$ machine table turning deflection [15]. 


\section{Surface Quality Prediction}

For this study, surface quality focuses on the surface flatness or profile. A detailed procedure is described for predicting the resultant surface flatness of a fixtured workpiece after machining. In general metal-cutting operations, the surface flatness of a machined part is affected mainly by clamping preloads, machining forces, fixturing rigidity, and machine tool stiffness. This analysis takes into account contributions from major effects, namely:

Fixture and machine table compliance.

Clamping distortion.

Workpiece deflection due to machining forces.

Forced vibration of the fixture-workpiece system.

\subsection{Deflection Due to Clamping Preload}

The clamping preload has a considerable impact on the accuracy of the final workpiece. When clamping preloads are applied, the workpiece is already deformed due to clamping forces prior to machining. The clamping forces also induce contact pressure on the interface between the workpiece and its locators/clamps. The deformations of contact regions result in datum-related errors, such as rigid body motions (slip and lift-off) of the workpiece, and hence change the positional accuracy of the features to be machined.

The clamping deformation of a workpiece being machined can be obtained from a FEA of the workpiece under the clamping forces and support conditions. Hence, the clamping deformation effect, on the surface flatness of the workpiece being machined, is computed by a separate FEA.

\subsection{Deflection Due to Machining Forces}

Instantaneous machining forces also have a significant impact on the surface accuracy of a machined workpiece. Owing to the machining forces, the workpiece, fixture, and machine tool may deflect. In this analysis, however, both the machine tool (except the machine table) and spindle are assumed to be rigid and only the effects of workpiece, fixture and machine table deflections are considered. Depending on the magnitude and direction of workpiece deflection, more or less (than the ideal machined surface) material may be removed, which results in a surface error on the workpiece.

In FEA, the cutting forces can be applied only to the nodal locations. Thus, the cutting force at any location is divided among the nodes of an element, using a shape function. The computation of workpiece deflection under machining forces is based on the flexibility influence coefficients, or compliance approach. This coefficient is defined as the static deflection at point $p$ owing to a unit force applied at point $q$, and is denoted as $d_{p q}$ [7]. When the machining forces and flexibility influence coefficients of the system are known, the instantaneous deflection of the workpiece owing to the machining forces can be calculated as the summation of the products of the magnitude of each machining force and the corresponding compliance coefficient at the point of application of the force.

Forced vibrations are caused by transient cutting forces and the repeated impacts of the cutting inserts when entering and exiting from the workpiece. The amplitudes of the forced vibrations can be characterised in terms of the dynamic stiffness of the fixture-workpiece system and cutting tools, which are often an order of magnitude lower than the corresponding static values. The transfer function or the frequency response function (FRF) is used to characterise the dynamic stiffness of a fixture-workpiece system. The real and imaginary parts of the transfer function are measured from the compliance, i.e. inverse of the stiffness, of the vibratory systems, in and out of phase of the applied machining forces. The amplitudes of vibrations increase if the frequency of the applied force, i.e. tooth passing frequency, matches one of the natural frequencies of the fixture-workpiece system. The machining process should ideally be operated away from these natural frequencies in order to keep the vibration levels low. Therefore, it is essential to be able to characterise the dynamic response of the workpiece as well as the fixture.

These kinds of dynamic data can be gathered using modal testing with the existing hardware or using an FEA of the fixture-workpiece system. In this study, NASTRAN (solution 103 ) is used to compute the compliance matrix based on the modal information. The FEA boundary conditions are similar to those applied for static and machining conditions. However, the fixture-workpiece contact effect is ignored in the dynamic mode analysis. An interactive Fortran program was developed to generate the dynamics data (real, imaginary, phase, and magnitude parts) for the FRF of any nodal point on the fixtureworkpiece FEA model. The inputs for this program are the NASTRAN result file ( $\sim$.pch), the damping ratio of the fixtureworkpiece system, the numbers of available modes (maximal 15 modes from the FEA result in this program), the excitation nodal point, and the response nodal point. The nodal points for excitation and response can be the same or different, depending on the input of the user. The frequency range of interest is also based on the specification of the user. Because the cutting force exerts on and excites a nodal point whose displacement (the response) will produce the surface error, the nodal points for excitation and response are the same as for the machining operation. Based on the type of machining operation, the directions $(x, y$, and $z$ ) for excitation and response can be the same or different.

According to the input data, the Fortran program calculates the vibration magnitude by extracting the real and imaginary parts for any specified nodal point from the FEA modal result. In this way, the FRFs in three directions $(x, y$, and $z$ ) for all the nodal points on a machined surface are computed. The natural frequencies of the fixture-workpiece system can be obtained from the FEA. The tooth passing frequency $(\mathrm{Hz})$ is defined as:

$$
T_{\text {freq }}=N \times r . p . m . / 60
$$

where $N$ is the number of teeth and r.p.m. is the rotational speed of the cutter. 


\subsection{Resultant Deflection of the Workpiece}

For an ideal machining process, the material to be removed will conform to the NC program. However, workpiece deflection caused by clamping preloads and cutting forces varies the depth of cut (DOC). When cutting forces are applied, the initial DOC can be reduced or increased according to the deflected shape of the workpiece. The true DOC then becomes the initial DOC which subtracts from or adds to the deflection of the workpiece. This gives rise to a variation of the true DOC along the surface and consequently a flatness error occurs. Furthermore, after releasing the clamps, the clamping deflection of the machined surface will disappear, affecting the machined surface accuracy. Forced vibration of the fixture-workpiece system is another factor contributing to the final machined surface error. The resultant surface error can be obtained by superimposing these three surface deflection components.

\section{Case Study}

A v-type production cast iron engine block and its fixture layout, shown in Fig. 3, is used as an example. The workpiece model consists of substructures (super-element model) and brick elements with elastic modulus $E=157500 \mathrm{MPa}$, Poisson's ratio $v=0.26$. The fixture layout applies a generic 42-1 locating scheme with four locating positions (two pins and two locators) on the primary datum and uses two top clamps. This cast iron workpiece surface contacts a spherical tip tool steel locator $(E=206800 \mathrm{MPa})$. Referring to Eq. (1) and assuming a locator diameter of $12 \mathrm{~mm}$, the deflection $y_{l}$ in $\mathrm{mm}$ is expressed as a function of load $P$ in Newtons as: $y_{l}=$ $1.6924 \times 10^{-4} P^{2 / 3}$.

The relationship of the locator spring force and the length change is shown in Fig. 4. Although this force-displacement relationship is a nonlinear curve, a linear spring with stiffness $k_{l}=1.594 \times 10^{5} \mathrm{~N} \mathrm{~mm}^{-1}$ can represent these locators approximately. $20 \mathrm{~mm}$ diameter screw clamps, which have a stiffness of $k_{c}=9.5 \times 10^{5} \mathrm{~N} \mathrm{~mm}^{-1}$ [12], are used to represent the clamping components. The applied clamping preload is $2700 \mathrm{~N}$ for each clamp (total $5400 \mathrm{~N}$ ).

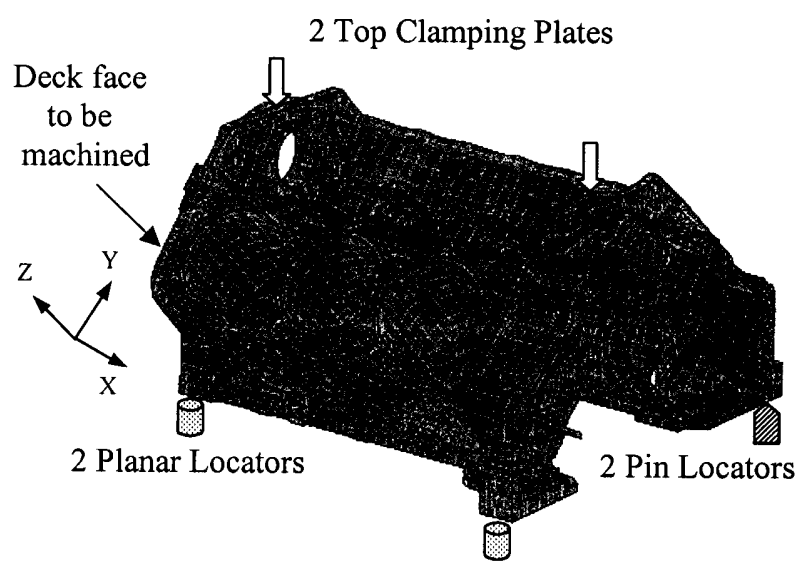

Fig. 3. Application example.

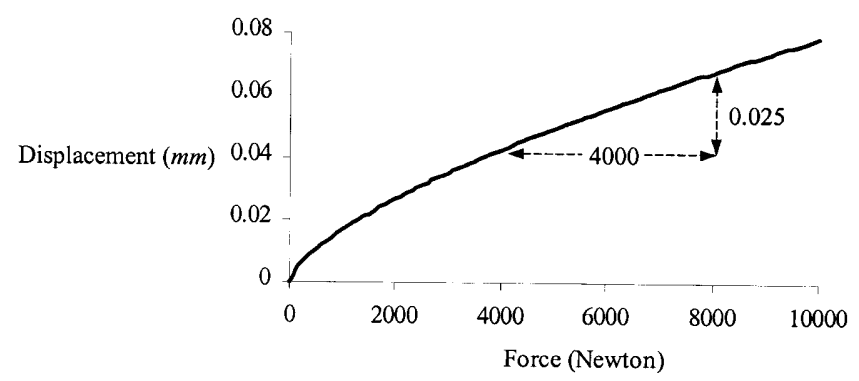

Fig. 4. Force-displacement curve of locator.

The modelling of fixture-workpiece contact interaction and fixturing boundary conditions affects the accuracy of the finiteelement results. The contact regions are modelled using surfacebased contact pairs. The clamp spring $K_{c}\left(9.5 \times 10^{5} \mathrm{~N} \mathrm{~mm}^{-1}\right)$ is connected to the other spring $K_{\text {top }}\left(5.7 \times 10^{5} \mathrm{~N} \mathrm{~mm}^{-1}\right)$ which represents the clamping structure. Similarly, the locator spring $K_{l}\left(1.594 \times 10^{5} \mathrm{~N} \mathrm{~mm}^{-1}\right)$ is connected to the other spring $K_{\text {tot }}\left(8.2 \times 10^{5} \mathrm{~N} \mathrm{~mm}^{-1}\right)$ which represents the fixture base $\left(K_{\text {fixel }}\right)$ and machine table $\left(K_{\text {mach }}\right)$. The spring constants of $K_{\text {top }}$ and $K_{f i x e l}$ are found from experiments using the modular fixture displayed in Fig. 1. The spring constant $K_{\text {mach }}$ is obtained from Fig. 2. This local modelling is illustrated in Fig. 5. Figure 5 (a) illustrates the local modelling of a workpiece and its locator. The workpiece and locator tip are modelled by finite elements and the locator-workpiece contact interaction is modelled as a surface-based contact pair. The locator tip FEA model is then connected to a spring element with stiffness of $K_{l}$, which represents the entire locator. Figure $5(b)$ shows that the clamping plate is modelled by a spring element to which the clamping preload is applied. In this study, the fixtureworkpiece contact interaction is taken into consideration when computing the clamping distortion. However, the deflections of a workpiece owing to machining forces ignore the contact deformation.

A face milling operation is performed to produce the finished deck surface of the engine block. The motion of the cutter is parallel to the $X$-axis, and moves in the positive direction (referring to Fig. 3). The tooling and cutting condition for this case are illustrated in Table 1. The magnitudes of cutting forces in three directions (tangential, radial, and axial) are predicted by a mechanistic cutting force model [16]. The

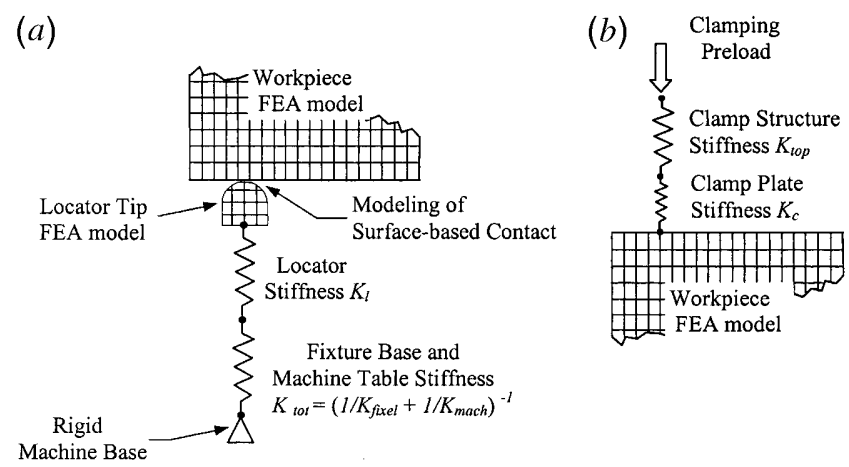

Fig. 5. Local modelling of $(a)$ the workpiece and its locator, and $(b)$ the workpiece and its clamp. 
Table 1. Cutting conditions and tooling geometry.

\begin{tabular}{|c|c|c|c|c|}
\hline Cutter diameter $\mathrm{mm}$ & Number of teeth & Radial rake & Axial rake angle & Lead angle \\
\hline 210 & 16 & $0^{\circ}$ & $5^{\circ}$ & $15^{\circ}$ \\
\hline Cutting conditions & & & Shape & Square \\
\hline D.O.C. $(\mathrm{mm})$ & 2.05 & Insert & I. C. $(\mathrm{mm})$ & 12.7 \\
\hline r.p.m. & 1340 & geometry & Material & Carbide \\
\hline Feed $\left(\mathrm{mm} / \mathrm{rev}^{-1}\right)$ & 2.16 & & Nose radius $(\mathrm{mm})$ & 2.36 \\
\hline
\end{tabular}

magnitude of the cutting force in the Z-direction (axial component of cutting force on the cutter) versus time is plotted in Fig. 6(a), and its power spectrum (in the frequency domain) is plotted in Fig. 6(b).

Figures 7 and 8 show the surface deflections due to clamping preloads and machining forces, respectively. The three components of machining forces (radial, axial, and tangential) causing deflections in the direction perpendicular to the machined surface are taken into account. The predicted workpiece deflection under clamping preloads lies in the range -2.63 to -0.78 $\mu \mathrm{m}$, and the deflection due to cutting forces lies in the range -9.53 to $4.88 \mu \mathrm{m}$. As illustrated in Fig. 8, the surface error is positive at the upper right corner, and negative on the lefthand side of the deck face. Thus, more (than intended) material is removed from the upper righthand corner compared to the lefthand side of the workpiece. Figure 9 illustrates the

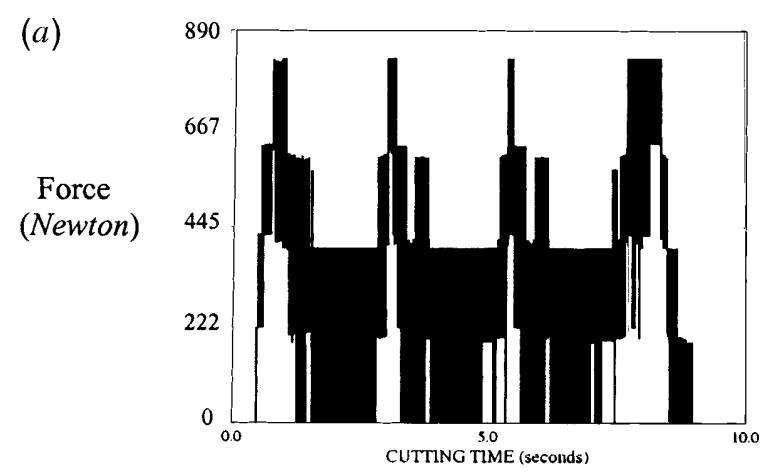

(b)

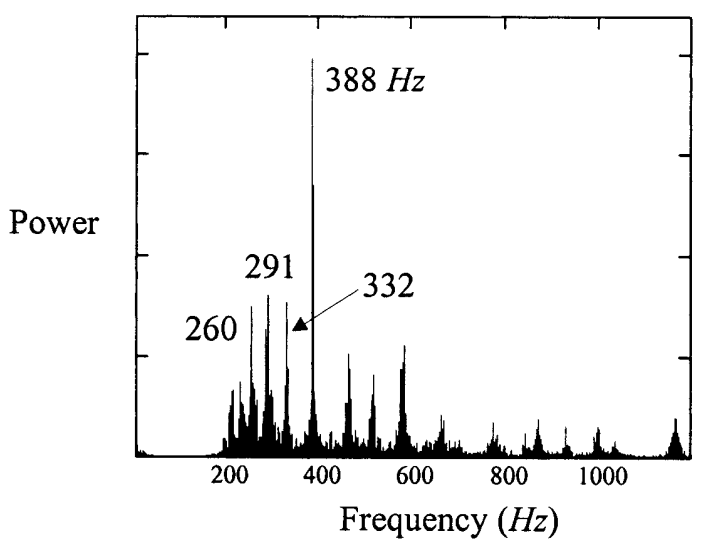

Fig. 6. (a) Axial cutting force versus time. (b) Spectrum of axial cutting force (in frequency domain).

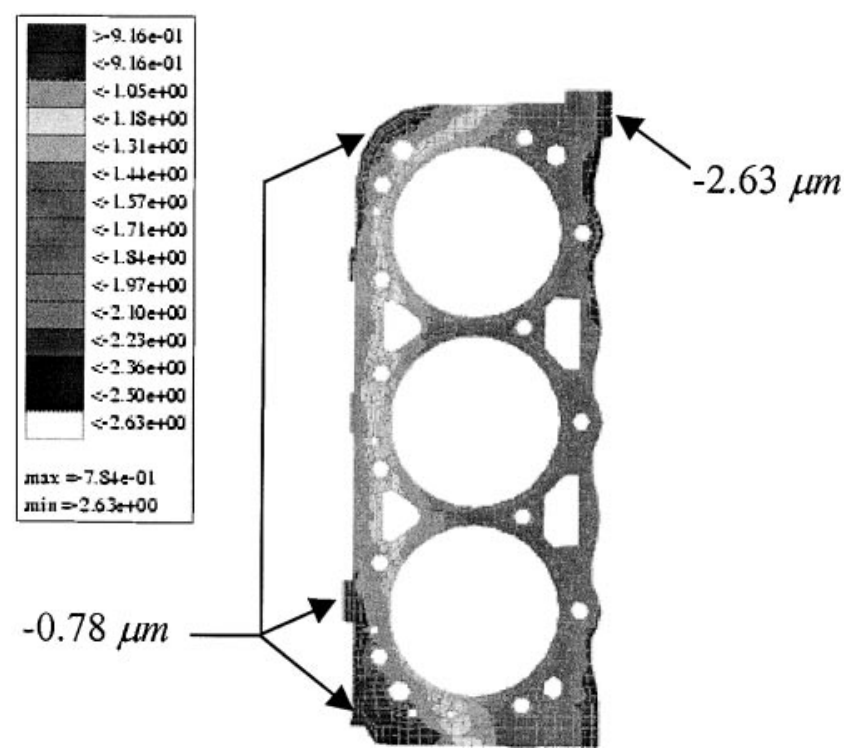

Fig. 7. Deflection due to clamping preloads. Deflection range: -2.63 to $-0.78 \mu \mathrm{m}$.

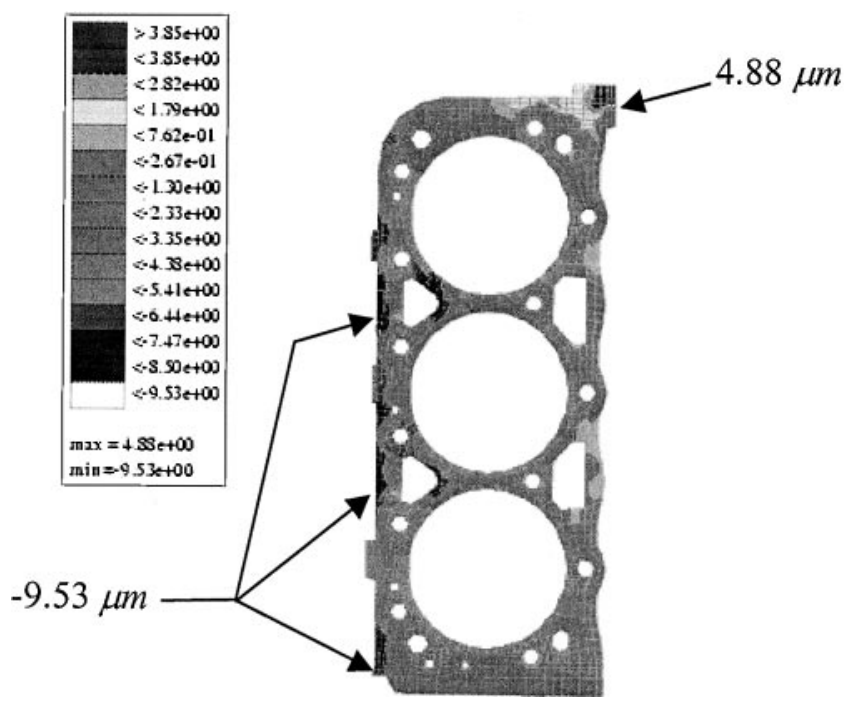

Fig. 8. Deflection due to cutting forces. Deflection range: -9.53 to $4.88 \mu \mathrm{m}$. 


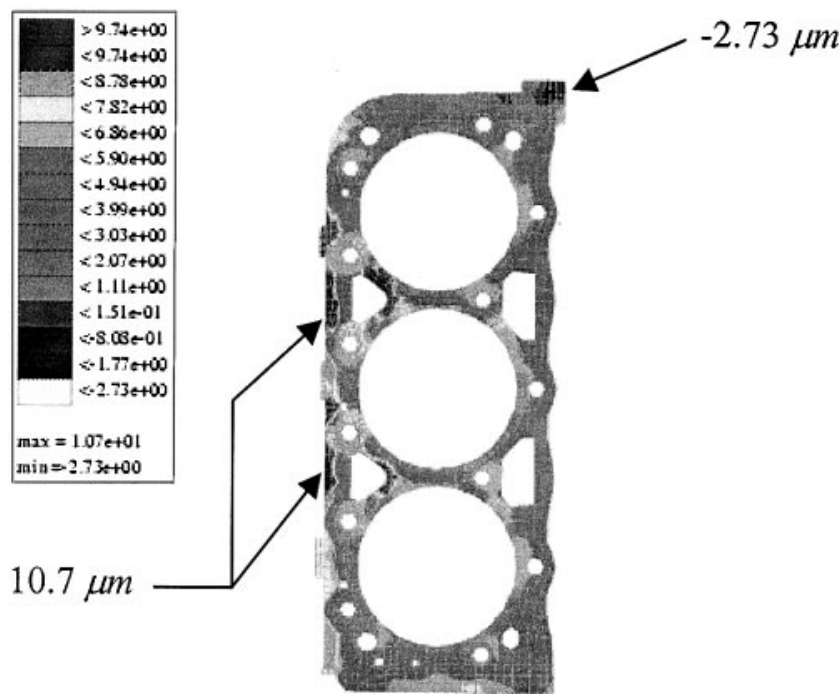

Fig. 9. Deflection after machining and unclamp. Deflection range: -2.73 to $10.7 \mu \mathrm{m}$.

resultant surface deflection after machining and releasing the clamps. After releasing the clamps, the workpiece elastic springback at the upper right is larger than that for the upper and lower left of the workpiece.

Another factor contributing to the machined surface error is the forced vibrations of the fixture-workpiece system. The lowest four natural frequencies obtained from FEA of this fixture-workpiece system are 828, 961, 1112, and $1240 \mathrm{~Hz}$. The tooth passing frequency is $357 \mathrm{~Hz}(16$ teeth $\times 1340$ r.p.m./60) which is very different from those natural frequencies. In a face milling operation, the axial cutting force generally has the greatest impact on the surface flatness. Therefore, the forced vibration is considered only in the direction perpendicular to the machined surface. The nodal points for excitation and response are the same when computing the vibration amplitude. The frequency range of interest is from $10 \mathrm{~Hz}$ to $3000 \mathrm{~Hz}$. The purpose of setting the lowest frequency $(10 \mathrm{~Hz})$ is to eliminate the fixture-workpiece rigid-body modes. The highest frequency is set at $3000 \mathrm{~Hz}$, which is much higher than the tooth passing frequency. The damping ratio is defined as 0.03 for the cast iron workpiece and steel fixture [17]. The FRF in the $\mathrm{z}$-direction (perpendicular to the machined surface) of several nodal points on the machined surface is displayed in Fig. 10, where the point numbers are referred to in Fig. 11. Vibration amplitudes, corresponding to the cutting forces at the tooth passing frequency, for all nodes on the machined surface are computed, as shown in Fig. 11.

From the simulation results, the deflection range of the final machined surface is from -2.73 to $12.68 \mu \mathrm{m}$, thus resulting in a surface flatness error of $15.41 \mu \mathrm{m}$ (peak-to-valley). This surface error is $14 \%$ due to clamping preloads and $80 \%$ due to machining forces. For machining deflection, axial cutting force (force component perpendicular to the machined surface) causes $53 \%$ of the surface deflection due to machining forces. Consequentially, the axial cutting force contributes $42 \%$ to the final surface error. Forced vibration contributes $6 \%$ to the final surface error. As illustrated in Figs 10 and 11, the middle

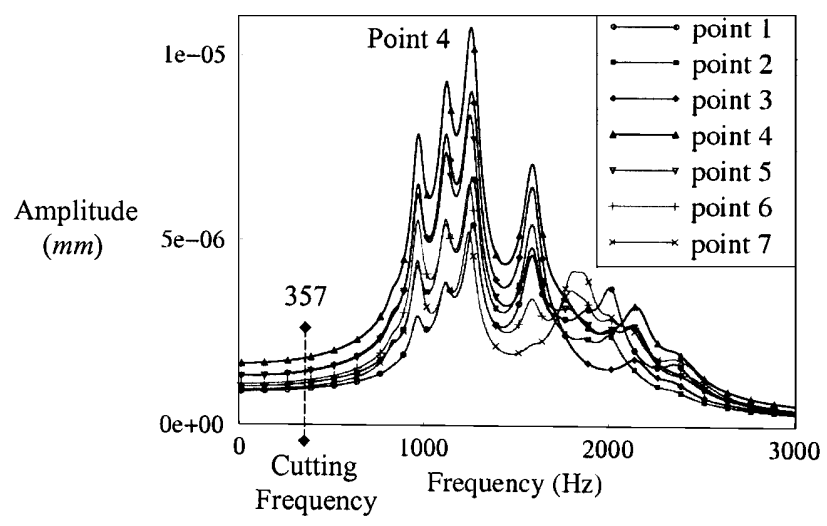

Fig. 10. FRF of sampled points on the machined surface (in the direction of the perpendicular to the machined surface).

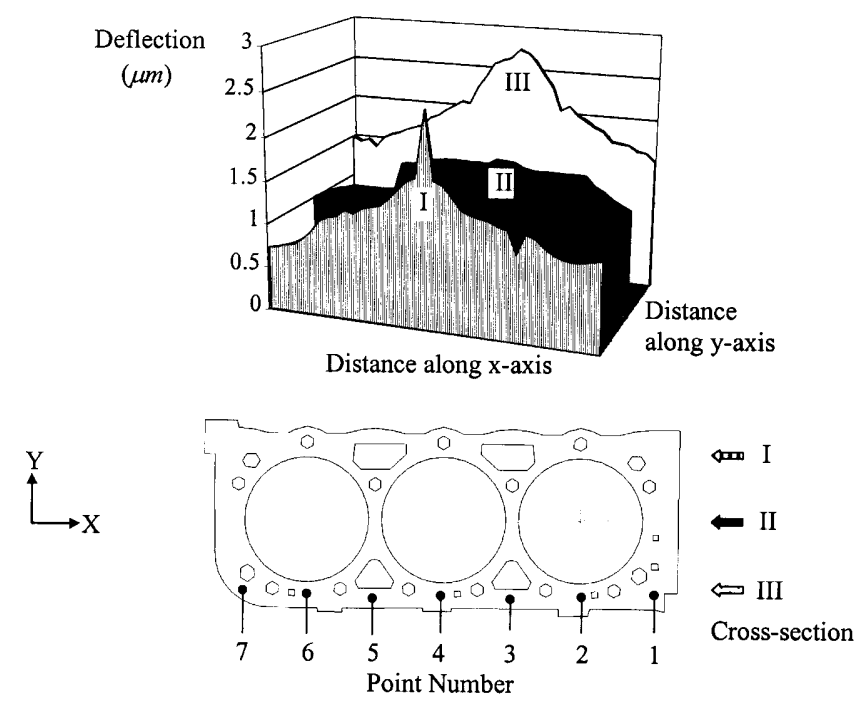

Fig. 11. Deflection due to forced vibration.

portion of the deck face (in the area of nodal point 4) has the highest vibration amplitude which is due to the structure of the workpiece and its fixturing scheme (the middle portion is away from the locator supports).

The quality of the finished part is checked by an off-line CMM (coordinate measuring machine). A Zeiss Vast CMM, as shown in Fig. 12, is used to measure the surface flatness of the engine block deck face. Three blocks are sampled from the same production line. Seventy-four points are measured on the machined deck surface for each block and the average flatness is $22.67 \mu \mathrm{m}$ for these sampled parts, as summarised in Table 2. A comparison between the experimental measurement and predicted data indicates that the simulation underestimated the experimental data. This underestimation is due mostly to the uncertainties in FEA, such as the assumptions of modelling and boundary conditions. Additionally, the effects of thermal expansion during machining and the machine spindle compliance are not considered. The difference between the predicted and measured peak-to-valley surface error values is about $32 \%$, which still provides a reasonable agreement. 


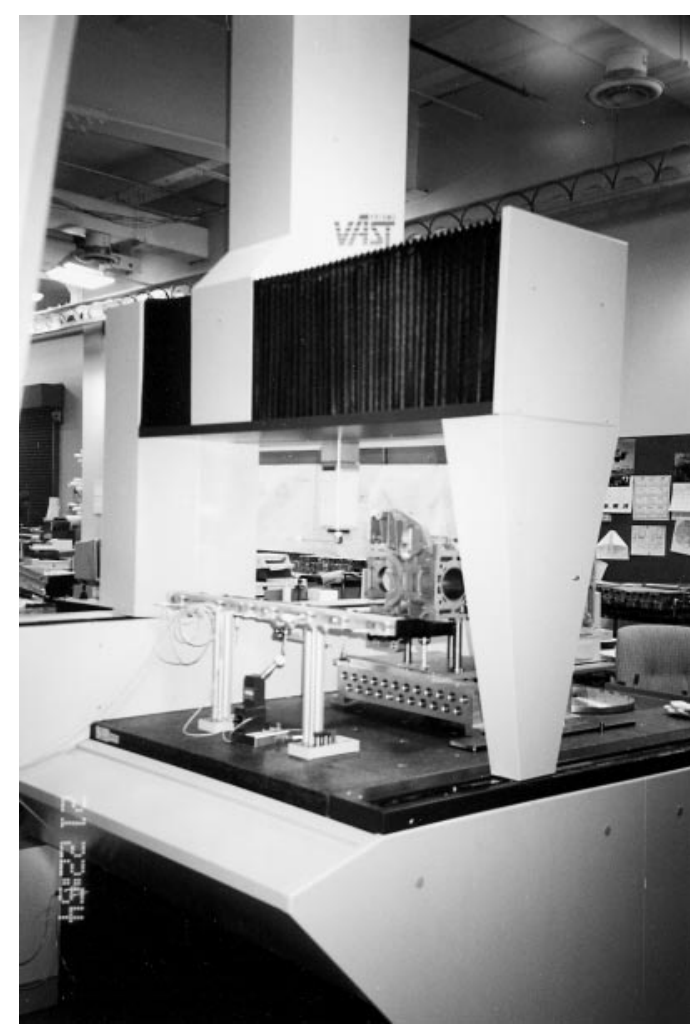

Fig. 12. Experimental measurement using CMM.

Table 2. Summary of experimental data (unit: $\mu \mathrm{m}$ ).

\begin{tabular}{lllll}
\hline $\begin{array}{l}\text { Block } \\
\text { number }\end{array}$ & Minimum & Maximum & $\begin{array}{l}\text { Flatness } \\
\text { (peak-to- } \\
\text { valley) }\end{array}$ & Specification \\
\hline 1 & -5 & 17 & 22 & \\
2 & -5 & 16 & 21 & $50 \mu \mathrm{m}$ \\
3 & -6 & 19 & 25 & \\
\hline
\end{tabular}

\section{Conclusions}

An integrated analysis model of the whole fixture-workpiece system is presented in this paper. For the purpose of machined surface quality prediction, a methodology is developed to integrate a FEA model of the locator-workpiece with the experimental stiffness of the fixture base and machine table. In this integrated model, the clamping preloads, machining forces, locator-workpiece contact interaction, fixture compliances, machine table stiffness, and forced vibrations, are taken into account. The benefits of this improved analysis model are a more realistic prediction of the machined surface quality and the provisions of a better understanding of the causes of surface error. Specifically,

The magnitude of surface error is linearly proportionally affected by the magnitudes of the external loads (clamping and machining forces). Based on the material, structure, and fixturing scheme of a workpiece, the clamping preloads and machining forces have different influences on the machined surface error. For a more rigid workpiece, such as a cast iron part in this example, the cutting forces have the greater (than clamping preloads) impact on the surface error.

The dynamic stiffness of a fixture-workpiece system depends on the structure of the workpiece and its locating and clamping arrangements. Hence, each machining feature on the workpiece has a different response to forced vibration. When the frequency of cutting forces (cutting tooth passing frequency) does not correspond with one of the natural frequencies of the fixture-workpiece system, the forced vibrations only slightly influence the final quality of a machined surface.

The final surface quality of the deck face for a production engine block is predicted. A comparison between the simulation result and experimental data shows a reasonable agreement. This application example demonstrates that the developed methodology is capable of estimating the surface quality for a large complicated workpiece.

\section{References}

1. J. D. Lee and L. S. Haynes, "Finite element analysis of flexible fixturing systems", Transactions ASME Journal of Engineering for Industry, 109, pp. 134-139, 1987.

2. R. J. Menassa and W. R. DeVries, "Optimization methods applied to selecting support positions in fixture design", Transactions ASME Journal of Engineering for Industry, 113, pp. 412-417, 1991.

3. G. Subramani, S. G. Kapoor and R. E. DeVor, "A model for the prediction of bore cylindricity during machining", Transactions ASME Journal of Engineering for Industry, 115, pp. 15-22, 1991.

4. N. N. Kakade and J. G. Chow, "Finite element analysis of engine bore distortions during boring operation", Transactions ASME Journal of Engineering for Industry, 115, pp. 379-384, 1993.

5. A. J. C. Trappey, C. S. Su and J. L. Hou, "Computer-aided fixture analysis using finite element analysis and mathematical optimization modeling", ASME Proceedings of International Mechanical Engineering Congress and Exposition, vol. 3(1), pp. 777787, 1995.

6. S. N. Melkote, S. M. Athavale, R. E. DeVor, S. G. Kapoor and J. J. Burkey, "Prediction of the reaction force system for machining fixtures based on machining process simulation", Transactions of NAMRI/SME, 23, pp. 207-214, 1995.

7. F. Gu, N. Melkote, S. G. Kapoor and R. E. DeVor, "A model for prediction of surface flatness in face milling", Transactions ASME Journal of Manufacturing Science and Engineering, 119, pp. 476-484, 1997.

8. P. Chandra, S. M. Athavale, S. G. Kapoor and R. E. DeVor, "Finite element based fixture analysis model for surface error predictions due to clamping and machining forces", ASME Proceedings of International Mechanical Engineering Congress and Exposition, vol 6(2), pp. 245-252, 1997.

9. ABAQUS/Standard User's Manual (Version 5.8), 2, HKS, Pawtucket, RI, 1998.

10. D. Bella and M. Reymond, (ed.), MSC/NASTRAN DMAP Module Dictionary, MacNeal-Schwendler, Los Angeles, CA, 1997.

11. J. H. Faupel and F. E. Fisher, Engineering Design - A Synthesis of Stress Analysis and Materials Engineering, 2nd edn, John Wiley, New York, pp. 811-816, 1981.

12. G. S. A. Shawki and M. M. Abdel-Aal, "Rigidity considerations in fixture design - rigidity of clamping elements", International 
Journal of Machine Tool Design and Research, 6, pp. 207$220,1966$.

13. Y. Liao, S. Hu and D. Stephenson, "Fixture layout optimization considering workpiece-fixture contact interaction: simulation results", Transactions of NAMRI/SME, 26, pp. 341-346, 1998.

14. Y. Zhu, S. Zhang and Y. Rong, "Experimental study on fixturing stiffness of T-slot based modular fixtures", Transactions of NAMRI/SME, 21, pp. 231-235, 1993.
15. M. A. Smith, "CNC machine tools performance evaluation", General Motors Corporation, Manufacturing Center Report, 1995.

16. F. Gu, S. Kapoor, R. DeVor and P. Bandyopadnyay, "An enhanced cutting force model for face milling with variable cutter feed motion and complex workpiece geometry", Transactions ASME Journal of Manufacturing Science and Engineering, 119, pp. 467-475, 1997.

17. C. M. Harris, Shock and Vibration Handbook, 4th edn, McGrawHill, New York, p. 43.13, 1996. 